\title{
Effects of cytomegalovirus infection in human neural precursor cells depend on their differentiation state
}

\author{
H. M. González-Sánchez • A. Monsiváis-Urenda • C. A. Salazar-Aldrete • \\ A. Hernández-Salinas • D. E. Noyola • M. E. Jiménez-Capdeville • \\ A. Martínez-Serrano • C. G. Castillo
}

Received: 18 August 2014 / Revised: 31 December 2014 / Accepted: 9 January 2015 /Published online: 8 April 2015

(C) Journal of NeuroVirology, Inc. 2015

\begin{abstract}
Cytomegalovirus (CMV) is the most common cause of congenital infection in developed countries and a major cause of neurological disability in children. Although CMV can affect multiple organs, the most important sequelae of intrauterine infection are related to lesions of the central nervous system. However, little is known about the pathogenesis and the cellular events responsible for neuronal damage in infants with congenital infection. Some studies have demonstrated that neural precursor cells (NPCs) show the greatest susceptibility to CMV infection in the developing brain. We sought to establish an in vitro model of CMV infection of the developing brain in order to analyze the cellular events associated with invasion by this virus. To this end, we employed two cell lines as a permanent source of NPC, avoiding the continuous use of human fetal tissue, the human SK-N-MC neuroblastoma cell line, and an immortalized cell line of human fetal neural origin, hNS-1. We also investigated the effect of the differentiation stage in relation to the susceptibility of these cell lines by comparing the neuroblastoma cell line with
\end{abstract}

H. M. González-Sánchez • C. A. Salazar-Aldrete •

M. E. Jiménez-Capdeville • C. G. Castillo $(\square)$

Department of Biochemistry, Facultad de Medicina, Universidad

Autónoma de San Luis Potosí, Av. Venustiano Carranza No. 2405,

Colonia Los Filtros, 78210 San Luis Potosí, SLP, México

e-mail: claudiacastillo@gmail.com

A. Monsiváis-Urenda

Department of Immunology, Facultad de Medicina, Universidad

Autónoma de San Luis Potosí, San Luis Potosí 78210, SLP, México

A. Hernández-Salinas $\cdot$ D. E. Noyola

Department of Microbiology, Facultad de Medicina, Universidad

Autónoma de San Luis Potosí, San Luis Potosí 78210, SLP, México

A. Martínez-Serrano

Department of Molecular Biology, Center of Molecular Biology

Severo Ochoa, Autonomous University of Madrid-CSIC Campus

Cantoblanco, Madrid, Spain the multipotent cell line hNS-1. We found that the effects of the virus were more severe in the neuroblastoma cell line. Additionally, we induced hNS-1 to differentiate and evaluated the effect of CMV in these differentiated cells. Like SK-N-MC cells, hNS-1-differentiated cells were also susceptible to infection. Viability of differentiated hNS-1 cells decreased after CMV infection in contrast to undifferentiated cells. In addition, differentiated hNS-1 cells showed an extensive cytopathic effect whereas the effect was scarce in undifferentiated cells. We describe some of the effects of CMV in neural stem cells, and our observations suggest that the degree of differentiation is important in the acquisition of susceptibility.

Keywords Cytomegalovirus $\cdot$ Neural stem cells $\cdot$ Neural progenitor cells $\cdot$ Cytophatic effect $\cdot$ Differentiation

\section{Introduction}

Human cytomegalovirus (HCMV), a member of the herpes virus family, is a common pathogen in immunocompromized patients and newborns. Excretion of viral particles in many body fluids, including urine and saliva, among others, is responsible for HCMV spreading which results in $40 \%$ seropositive adults in developed countries. The incidence of infection in developing countries is even higher, where almost $100 \%$ of the population has been infected by adulthood (Wynn and Khanna 2006; Ornoy 2007; Noyola et al. 2010). Congenital infection by HCMV has an incidence between 0.8 and $2.6 \%$ worldwide, and this infection is the most frequent cause for developmental brain disorders, including mental retardation and hearing loss.

Although most of the research about pathogenic mechanisms of CMV in the brain has been performed on animals, 
an important limitation has been the strict host specificity of CMVs, which requires the utilization of the corresponding animal virus (Wynn and Khanna 2006). Furthermore, the clinical manifestations on some animal models are different from those observed in the human central nervous system (CNS) (Woolf et al. 2007). Nevertheless, these animal models have provided evidence that the highest CMV susceptibility in CNS is presented by neural precursor cells (NPCs) (Tsutsui et al. 2002). Infection of these cells might be the cause of microcephaly and mental retardation since it has been observed that murine CMV inhibits NPC proliferation and migration (Shinmura et al. 1997; Tsutsui et al. 2008). Furthermore, studies of functional neural cells after CMV infection, though scarce, reveal that infected cells have altered intercellular communication and deficient response to neurotransmitters (Ho and van den Pol 2007).

In order to study NPC in vitro, it is necessary to isolate them from embryonic tissue and maintain them in culture under specific conditions to favor neural proliferation. In vitro studies in which these cells were infected with human CMV demonstrated that NPCs are highly permissive to CMV infection and all types of viral proteins are expressed. In addition, cytopathic effect (CPE) in these cells at the multiplicity of infection (MOI) of three plaque formation units (PFU) per cell consisted in formation of syncytia, which can be the result of viral cell to cell dissemination (Luo et al. 2008). Infection of these cells may increase the apoptosis frequency and inhibit neuronal and glia differentiation; however, this effect remains controversial since it has also been reported that CMV infection may cause a spontaneous, premature, and abnormal NPC differentiation (Odeberg et al. 2006, 2007; Luo et al. 2010).

Recently, the importance of the differentiation degree related to the capacity of the virus to effectively infect the cells during the first steps of the viral cycle was described. It was shown that the concentration of heparan sulfate in the cell surface, the presence of $\beta 1$-integrin and vimentin, and the nuclear pore density is important for the attachment, penetration, and internalization to the nucleus (Kawasaki et al. 2011). In mouse models, it has been observed that embryonic stem cells cannot be infected with CMV and they acquire susceptibility through differentiation (Matsukage et al. 2006; Kawasaki et al. 2011). However, differentiated neurons are also refractory to the infection and this may suggest that only during a precise period during differentiation, the cells can be infected (Cheeran et al. 2005).

Taking this into account, we aimed to establish an in vitro model of CMV infection in NPC that could help clarify these issues. In order to do so, we employed, as a permanent source of NPC, two cell lines, avoiding the continuous use of human fetuses, the human SK-N-MC neuroblastoma cell line and an immortalized cell line of fetal origin hNS-1. The human neural stem cell line hNS-1 was isolated from a 10.5 -week-old human fetal tissue, immortalized using a retroviral vector encoding v-myc fusion protein, and they proliferate in the presence of growth factors (EGF and bFGF) (Villa et al. 2000, 2004). These cells are multipotent, and upon removal of growth factors, they readily differentiated into neurons $(\beta$ tubulin III $+14.3 \pm 1.35 \%$ ), astrocytes (glial fibrillary acidic protein $($ GFAP),$+ 33.4 \pm 1.85$ ), and oligodendrocytes (Gal C $<1 \%$ ) (Martinez-Serrano et al. 2001; NavarroGalve et al. 2005; Rubio et al. 2000; Villa et al. 2000, 2001, 2002). We also investigated the effect of the differentiation degree related to the susceptibility of these cell lines by comparing the neuroblastoma cell line with the multipotent cell line hNS-1. We found that the effects of the virus were more severe in the neuroblastoma cell line. Additionally, we induced hNS-1 to differentiate and evaluated the effect of HCMV in these differentiated cells. Like SK-N-MC cells, hNS-1differentiated cells were also susceptible, although not in the same extent. We describe some effects of HCMV in NPC, showing the importance of the differentiation degree in the acquisition of susceptibility.

\section{Methods}

Cell culture and media The human NPC cell line hNS-1 was generously donated from Centro de Biología Molecular "Severo Ochoa," Madrid, Spain. These cells were grown in poly-L-lysine (Sigma-Aldrich, St. Louis, MO)-coated plastic flasks using a cell culture medium that contained DMEM-F12 (1:1, Gibco, Invitrogen Life Technologies, Carlsbad, CA) supplemented with $0.6 \%$ glucose, $\mathrm{N} 2$ (Life Technologies), $0.5 \%$ AlbuMAX I (Gibco), and human epidermal and fibroblast growth factors (EGF/FGF-2, R\&D Systems) as previously described (Villa et al. 2000). For differentiation experiments, hNS-1 cells were cultured 5 days without FGF- 2 and EFG and in the presence of fetal bovine serum $0.5 \%$ (FBS, ATCC, Rockville, MD). SK-N-MC cells (ATCC, Lot 4399800, Manassas, VA) were grown in MEM (ATCC) supplemented with $10 \%$ FBS. Human foreskin fibroblasts were obtained from primary culture and were grown in DMEM medium supplemented with $10 \%$ calf serum (Gibco). MDCK cells (ATCC) were grown in the same conditions as fibroblasts. Penicillin and streptomycin were used $(100 \mathrm{U} / \mathrm{mL}$ and $100 \mu \mathrm{g} / \mathrm{mL})$ to prevent culture contamination. For cells requiring serum, a maintenance medium was used where the serum concentration was decreased to $2 \%$ after CMV inoculation. All cell cultures were grown at $37{ }^{\circ} \mathrm{C}$ in a $95 \%$ humidity and a $5 \%$ $\mathrm{CO}_{2}$ atmosphere. Cell karyotyping and mycoplasma contamination were assessed periodically.

Virus strain and infection Human CMV AD169 laboratory strain was propagated in fibroblast cultures; cell-free viral stocks were obtained from supernatants, frozen, and stored 
at $-70{ }^{\circ} \mathrm{C}$ until use. Viral titers were determined by plaque assay as previously described (Wentworth and French 1970). Real-time quantification (LightCycler CMV LC Set vers 2.0) of the viral stock was carried out, and cell lines and fibroblasts were serum-starved and infected by virus adsorption for 2 to $24 \mathrm{~h}$; then, maintenance medium was added. For differentiation experiments, infection was carried out after 5 days of growth factor starving.

Experimental design In all experimental approaches, the 0.01 MOI was administrated and a lower or a higher MOI, depending on the technique, was employed. In order to monitor CMV infection in NPC, we followed three procedures. First, we periodically analyzed morphological changes through light microscopy observation after cell infection at 0.01 and 0.1 MOI of CMV for $2 \mathrm{~h}$. In addition, we confirmed infection using a nested PCR to determine viral production in NPC (SK-N-MC and hNS-1); we used MDCK and fibroblasts as negative and positive controls of viral production, respectively. In this case, we employed $2 \mathrm{~h}$ of infection with 10 DNA viral copies per cell and supernatant aliquots of these infected cells were drawn at 1, 4, 7, 11 and 14 days post-infection (dpi) to test for the presence of CMV through PCR. Finally, we demonstrated the presence of CMV within the infected cells ( $0.1 \mathrm{MOI}$ of CMV for $24 \mathrm{~h}$ ) by detection of the viral glycoprotein $\mathrm{B}(\mathrm{gB})$ by immunocytochemistry at $7 \mathrm{dpi}$. In this case, a higher MOI was used because of the low detection of positive cells. We also analyzed cellular viability by trypan blue assays and estimated proliferation using 3-(4,5-dimethylthiazol-2-yl)-5-(3-carboxymethoxyphenyl)-2-(4sulfophenyl)-2H-tetrazolium, inner salt (MTS)/phenazine methosulfate (PMS) assay (Promega, Fitchburg, WI) at 1, 4, and $7 \mathrm{dpi}$. NPC and fibroblasts were infected at 0.01 and 0.001 MOI of CMV for $24 \mathrm{~h}$, controls corresponded to mockinfected cells, and cisplatin (Sigma-Aldrich)-intoxicated cells were used as a death control and inhibitor of proliferation (2.5 $\mu \mathrm{M}$ for NPC and $50 \mu \mathrm{M}$ for fibroblasts). Additionally, carboxyfluorescein (CFSE, Invitrogen) assay was used to analyze proliferation at 1,4 , and $7 \mathrm{dpi}(0.01 \mathrm{MOI})$.

CPE on NPC Generally, the term CPE is attributed to diverse pathological alterations in cells triggered by eukaryotic viruses (Agol 2012). It includes the ability to injure or kill the infected cells. In the case of CMV, intranuclear inclusions described like a central nuclear body surrounded by a halo termed owl's eyes are the typical CPE (Ho 2008). In order to identify the CPE, cells were seeded at $1 \times 10^{5}$ cells/well and, after attachment, were infected for $2 \mathrm{~h}$ and kept in maintenance medium until 10-14 dpi. Cells were observed to evaluate morphological changes including cytomegalic inclusions (owl's eyes), vacuoles, cellular size changes, cellular soma granulations, nuclear appearance variation, and the length of cellular prolongations. We analyzed at least five random fields per well using an inverted microscope Nikon TMS at $\times 10$ and $\times 20$ magnifications and performed at least three independent experiments.

Immunocytochemical evaluation NPC $\left(5 \times 10^{4}\right.$ cells/well $)$ were seeded in coverslips, and after attachment, they were infected with 0.01 MOI of CMV. Coverslips were washed with phosphate-buffered saline (PBS) and fixed with paraformaldehyde $4 \%$ for $10 \mathrm{~min}$; they were washed three times with a washing buffer ( $1 \%$ FBS and $0.025 \%$ Triton X-100 in PBS) for $10 \mathrm{~min}$ and sodium borohydride (Sigma-Aldrich) for $10 \mathrm{~min}$, followed by three washes with the washing buffer and incubation with a blocking buffer $(10 \% \mathrm{FBS}, 0.025 \%$ Triton X-100 in PBS) for $2 \mathrm{~h}$, and left overnight at $4{ }^{\circ} \mathrm{C}$ with the mouse monoclonal primary antibodies against viral gB (1:800, Serotec, Raleigh, NC), nestin (1:1000, Santa Cruz Biotechnology, Dallas, TX), and microtubule-associated protein 2 (MAP-2) (1:1000, Sigma) or with the rabbit polyclonal primary antibody against GFAP (1:1000, Dako, Mexico, DF). Coverslips were washed three times with the washing buffer and incubated with the polyclonal goat anti-mouse Cy3-conjugated (1:300, Abcam), rabbit anti-mouse FITC-conjugated (1:300, Serotec) or goat anti-rabbit Alexa-546-conjugated (1:300, Invitrogen) antibodies for $2 \mathrm{~h}$. Cell nuclei were counterstained with Hoechst 33258 $(0.2 \mu \mathrm{g} / \mathrm{mL}$, Invitrogen) or propidium iodide ( $5 \mu \mathrm{g} / \mathrm{mL}$, Sigma) for $5 \mathrm{~min}$. Microscopic examination and photography of specimens were performed in a Olympus BX41 epifluorescence microscope. The quantification of CMV-infected cells was carried out in a BD FACSCalibur flow cytometer by detecting the percentage of gB-immunoreactive cells.

Viral production assay Onto six-well plates, $2 \times 10^{5}$ cells/well were seeded, supernatant aliquots (two wells for each study condition, $200 \mu \mathrm{L}$ each) were taken, and cells were infected. After $2 \mathrm{~h}$ of incubation, cells were washed with PBS and $4 \mathrm{~mL}$ of maintenance medium was added. Aliquots were taken at different dpi and preserved at $-70{ }^{\circ} \mathrm{C}$ until use. Medium reposition was done immediately before each sample was obtained. Cells were trypsinized at $14 \mathrm{dpi}$ and were processed with the supernatants. Each sample was spiked with $5 \times 10^{4}$ SK-N-MC cells to homogenize the actin control. DNA extraction and nested PCR (described below) were carried out, and electrophoretic products were photographed and analyzed by densitometry with the program ImageJ (Wayne Rasband, HIMH, NIH, free access http://rsbweb.nih.gov/ij/).

Nested PCR Viral DNA was extracted from culture supernatants $(0.2 \mu \mathrm{L})$ as previously described (Monsivais-Urenda et al. 2010). Outer (forward 5'-GTC AAA CAG ATT AAG GTT CGA GTG G-3', reverse 5'-TGT ACT CAT TAC ACA TTG TTT CCA CAC-3') and inner (forward 5'-ACT GGC GCC TTT AAT ATG ATG GG-3', reverse 5'-GAG CAC TGA GGC AAG TTC TGC-3') primers were aimed to the immediate early protein 1 gene of CMV and amplified a final 
380-bp fragment (Monsivais-Urenda et al. 2010). Another set of primers (forward 5'-ACG GCT CCG GCA TGT GCA AG$3^{\prime}$ and reverse $5^{\prime}$-TGA CGA TGC CGT GCT CGA TG-3') were used in the same reactions as an internal control which amplified $\beta$-actin gene ( 240 pb, Köhler 1995). For both PCRs, $0.06 \mathrm{UI} / \mu \mathrm{L}$ Taq (Vivantis Technologies Sdn Bhd, Selangor DE, Malaysia) was utilized in a final volume of $12.5 \mu \mathrm{L}$ per reaction. In the first PCR, $0.5 \mu \mathrm{mol} / \mathrm{L}$ of CMV outer primers, $0.075 \mu \mathrm{mol} /$ $\mathrm{L}$ of $\beta$-actin primers, and $1 \mu \mathrm{L}$ of DNA were used, whereas in the second reaction, $0.5 \mu \mathrm{mol} / \mathrm{L}$ of $\mathrm{CMV}$ inner primers, $0.1 \mu \mathrm{mol} / \mathrm{L}$ of $\beta$-actin primers, and $1 \mu \mathrm{L}$ of the first reaction were employed. PCR conditions were the same for both reactions, beginning with DNA denaturalization at $94{ }^{\circ} \mathrm{C}$ for $5 \mathrm{~min}$ and 30 amplification cycles $\left(94{ }^{\circ} \mathrm{C}\right.$ for $1 \mathrm{~min}, 60^{\circ} \mathrm{C}$ for $1 \mathrm{~min}$, and $72{ }^{\circ} \mathrm{C}$ for $1 \mathrm{~min}$ ) followed by a final extension of $10 \mathrm{~min}$ at $72{ }^{\circ} \mathrm{C}$. Nested PCR products were observed after electrophoresis in a $1.5 \%$ agarose gel stained with ethidium bromide and photographed using a Kodak Gel Logic 100 transilluminator. The detection limit of this assay is $1 \times 10^{4}$ DNA copies $/ \mathrm{mL}$.

Trypan blue assay NPC and fibroblasts were seeded at $5 \times 10^{4}$ cells/well in 24-well plates. After attachment, the cells were infected with CMV or exposed to cisplatin for $24 \mathrm{~h}$. Viability assays were performed using $0.05 \%$ trypan blue in a cellular suspension of $1 \mathrm{~mL}$. After incubation for $5 \mathrm{~min}$, dead (blue) and live (colorless) cells were counted in a Neubauer chamber. The percentage of viable cells was obtained from four replicates of each condition, using three independent experiments.

MTS/PMS assay The proliferation assay was performed using a kit which determines the overall amount of metabolically active cells through conversion of a tetrazolium salt to formazan which is solubilized and determined spectrophotometrically at $490 \mathrm{~nm}$. In 96-well plates, $5 \times 10^{4} \mathrm{NPC}$ and $2.5 \times 10^{4}$ fibroblasts were seeded, and after attachment, the cells were infected with $\mathrm{CMV}$ or exposed to cisplatin. At 1 and $7 \mathrm{dpi}, 20 \mu \mathrm{L}$ of a MTS/PMS mix was added to each well and incubated at $37^{\circ} \mathrm{C}$ for $2 \mathrm{~h}$ for NPC and $1 \mathrm{~h}$ for fibroblasts, and then, absorbance was measured in a BioTek ELx800 spectrophotometer. Metabolic activity percentage was calculated through comparison with the average absorbance of the mock-infected control. At least four replicates were performed for each condition in three independent experiments.

CFSE assay NPCs were seeded at $2 \times 10^{5}$ cell/well in six-well plates. Culture medium was removed, and $500 \mu \mathrm{L}$ of PBS and $1 \mu \mathrm{L}$ of CFSE $(5 \mathrm{mM})$ were added. Cells in these conditions were incubated in the dark for $10 \mathrm{~min}$, and after this time, $2 \mathrm{~mL}$ of medium was added and removed immediately and cells were maintained in incubation with growth medium until the indicated times. Then, at the indicated days, cells were trypsinized and suspended in p-formaldehyde $1 \%$ and analyzed in a BD FACSCalibur flow cytometer.
Statistical analysis Data were expressed as mean \pm standard deviation (SD) or median \pm interquartile range (IQR). If the data meet the specifications, parametric assays were performed, Student $t$ test or ANOVA for multiple comparisons with Bonferroni post hoc test. In order to analyze nonparametric data, Mann-Whitney $U$ was used. A level of $<0.05$ was considered significant. All data were analyzed in GraphPad Prism 5.01.

\section{Results}

Productive infection rate in SK-N-MC is higher than in hNS-1 cells

In order to demonstrate HCMV protein expression in NPC with the AD169 HCMV strain, infected cells in coverslips were stained for the presence of the late viral $\mathrm{gB}$ at $7 \mathrm{dpi}$. In addition, nestin antibody was used to show the NPC identity (Fig. 1a). Quantification of the percentage of gBimmunoreactive cells at 7 dpi showed that SK-N-MC cells $(95.4 \pm 2.7 \%, n=4)$ are much more susceptible to be infected with HCMV than hNS-1 cells $(8.4 \pm 4.5 \%, n=7)$ at an MOI of 0.1 (Fig. 1b). As gB results from HCMV late gene expression, this assay is an indirect verification of viral immediate-early and early gene expression, since viral replication was efficient and complete viral particle formation was inferred in these cells. Additionally, we sought to investigate if these NPCs can produce viral particles, so we performed a comparative assay using fibroblasts in which viral propagation in vitro normally occurs (positive control). On the other hand, MDCK cells were used as a negative control of viral production based on its canine origin. The media from such cells were collected at different dpi, and viral production was quantified by a nested PCR. As we expected, in MDCK cells, viral production was null (not shown), whereas SK-N-MC cells followed the same behavior as fibroblasts. Even though viral production was slightly lower at $1 \mathrm{dpi}$, it was similar to that of fibroblasts in the last time points studied. On the contrary, viral production was almost null in hNS-1-infected cells, showing that these can be infected but they cannot actively replicate this virus (Fig. 1c).

\section{CPE of human CMV in NPCs}

After HCMV infection in SK-N-MC cells, we observed an important cellular loss and the development of viral concentration-dependent CPE. In these cells, vacuole formation and presence of granulation were not as evident as they were seen during fibroblast infection (not shown). SK-N-MC cells infected with $0.1 \mathrm{MOI}$ initially showed a loss of normal morphology, since their multiple short-length cytoplasmic 
Fig. 1 Viral production in SK-N$\mathrm{MC}$ is higher than in hNS-1 cells. a Cells were seeded in coverslips, fixed, and processed for immunofluorescence using Abs for nestin, a NPC marker (nuclei were counterstained with Hoechst 33258) and for gB, a HCMV late protein at 7 dpi with HCMV 0.1 MOI (nuclei were counterstained with propidium iodide). Scale bar, $50 \mu \mathrm{m}$. b Using flow cytometry, the numbers of gB-positive cells were quantified at $7 \mathrm{dpi}$ with $0.1 \mathrm{MOI}$ (mean percentage $\pm \mathrm{SD}$, $n=$ at least three independent experiments, $* * * p<0.001 t$ test). c NPC and fibroblasts were infected with 10 viral DNA copies/mL, and supernatants were collected at $1,4,7,11$, and 14 dpi. Increase in viral genetic material during infection progression is shown $\left({ }^{*} p<0.05,{ }^{* *} p<0.01\right.$, $* * * p<0.001$ vs $1 \mathrm{dpi}$ for each cell line). Each point represents median of two independent experiments with at least three replicates, and error bars correspond to interquartile range

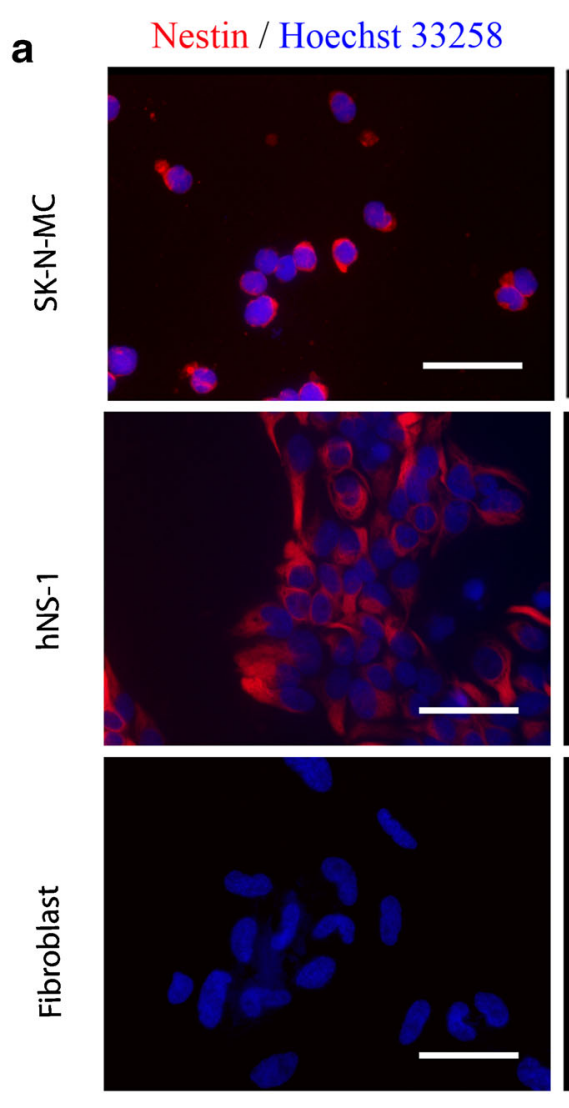

gB/ Propidium iodide
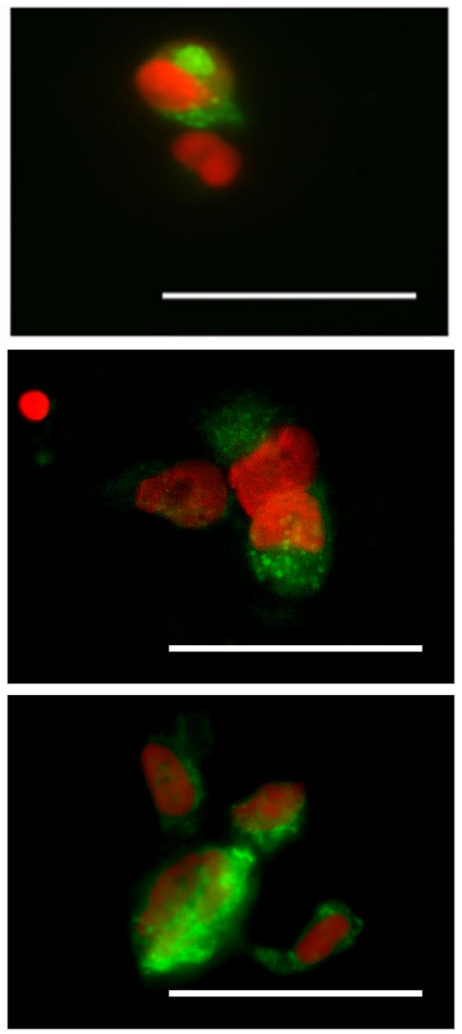

\section{b}

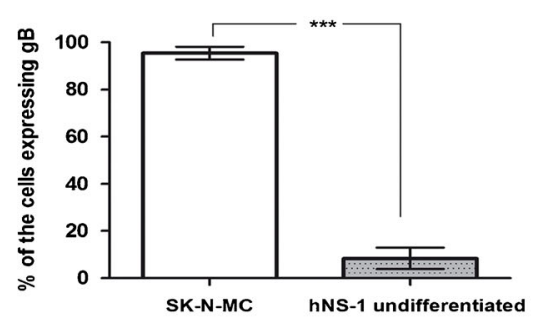

C Viral production in neural precursor cells
and fibroblast

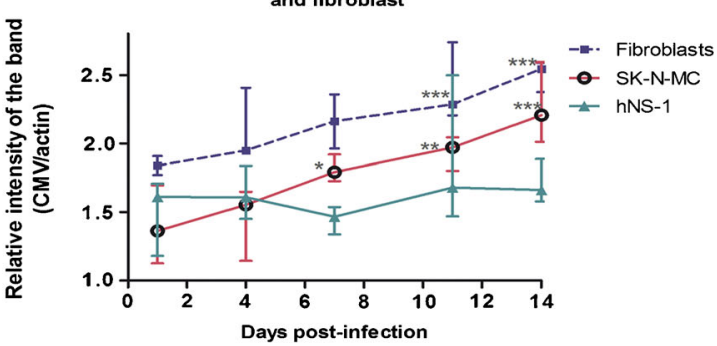

prolongations were lost (Fig. 2a) and the cells turned to a rounded or fusiform shape after infection. At this viral concentration, the predominant effect was the formation of syncytia that are described as multinuclear giant cells; these increased in number and size as the infection progressed (Fig. 2b, arrows). In some cases, we found intracellular inclusions characterized by a rounded nucleus surrounded by a halo, which are known as owl's eyes (Ho 2008) (Fig. 2b, arrowheads). The CPE of SK-N-MC cells infected with 0.01 MOI was very different. Starting on $4 \mathrm{dpi}$, some cells exhibited cytoplasmic prolongations that suggest neural differentiation. An increasing number of cells were observed as HCMV infection progressed (Fig. 2c). At 10 dpi, however, the number of this kind of cells decreased and syncytia formation was noticed as had been observed with the other concentration (not shown). Conversely, during HCMV infection of hNS-1 cells, we did not observe changes neither at $0.1 \mathrm{MOI}$ nor at
0.01 MOI; only slight syncytia formation and scarce owl's eyes at advanced dpi were observed (Fig. 2d-f).

Viability of SK-N-MC decreases during CMV infection whereas hNS-1 cells are resistant

Mock-infected and HCMV-infected cells were analyzed for cellular viability in a trypan blue assay at 1 and 7 dpi. In SK-N-MC cells, an increased cell death was demonstrated with $0.01 \mathrm{MOI}$ at $1(68.8 \pm 7 \%, p<0.05)$ and $7 \mathrm{dpi}(61.4 \pm$ $20 \%, p<0.05$ ), whereas with $0.001 \mathrm{MOI}$, any significant effect in cellular viability was noticed $(73.7 \pm 3 \%$ at $1 \mathrm{dpi}$ and 71 $\pm 16 \%$ at 7 dpi) when compared against mock-infected cells $(84.15 \pm 2 \%$ at $1 \mathrm{dpi}$ and $90.9 \pm 4 \%)$. On the other hand, hNS1 cells appeared to be resistant to CMV infection since we did not observe any change in cellular viability with any of the viral doses used (Fig. 3a). 


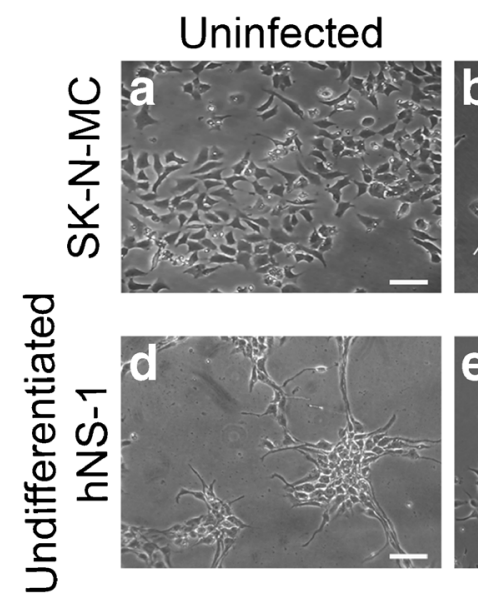

Fig. 2 Cytopathic effect of HCMV in NPC. Pictures shown are representative of image series of SK-N-MC or hNS-1 cells mockinfected or after infection with $0.01 \mathrm{MOI}$ or $0.1 \mathrm{MOI}$ at 7 dpi. a-c In SK-N-MC cells, the predominant effect with 0.01 MOI was cytoplasmic prolongations, whereas after infection with the higher viral concentration,

Infected NPCs increased their metabolic activity early during infection and modify proliferation according to the infection progress

In order to assess if HCMV-infected NPCs have an effect in proliferation, SK-N-MC and hNS-1 cells were either infected with 0.01 and 0.001 MOI of HCMV or mock-infected. Cellular proliferation was quantified 1 and 7 dpi by a MTS/PMS assay. We found a significant impact of CMV infection on SK$\mathrm{N}-\mathrm{MC}$ cells. At $1 \mathrm{dpi}$, we observed that cellular proliferation increased in infected cells compared with that in mock-
MOI 0.1
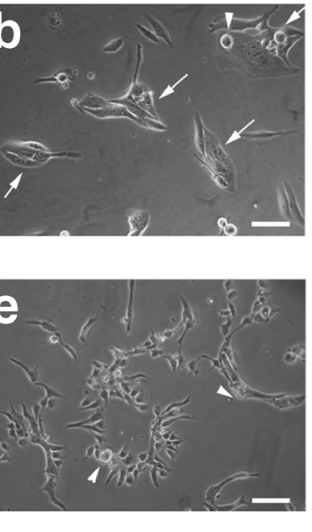

we observed syncytia formation (arrows) and cytoplasmic inclusions (arrowheads). d-f In hNS-1, we did not observe any evident change, only slight syncytia formation and scarce cytoplasmic inclusions. Scale bar, $50 \mu \mathrm{m}$

infected cells ( $117.1 \pm 12$ and $115.2 \pm 1.4 \%$ activity related to control for 0.001 and $0.1 \mathrm{MOI}$, respectively). Nevertheless, this effect was reversed at 7 dpi when a clear proliferation reduction was observed and it was even more pronounced with the higher viral concentration $(56.2 \pm 3.7 \%$ for MOI of 0.001 and $30 \pm 11 \%$ for MOI of $0.01, p<0.001$ ) (Fig. 3b). In the case of hNS-1 cells, we did not observe any change in proliferation even with the highest viral concentration employed (Fig. 3b). These contrasting results could reflect the difference in susceptibility between these two NPCs. To confirm that these changes indeed reveal the effect in cellular
Dpi 1
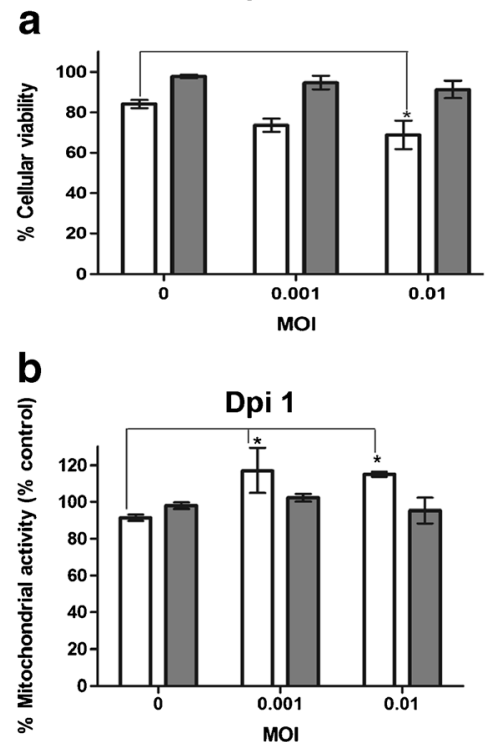

Fig. 3 Viability of SK-N-MC decreases during HCMV infection whereas hNS-1 cells are resistant. SK-N-MC and hNS-1 cells were infected with MOI of 0.01 and 0.001 or mock-infected. a Trypan blue assay was performed at 1 and 7 dpi (mean percentage $\pm \mathrm{SD}, n=4$ ). b Metabolic activity was analyzed at 1 and 7 dpi using a colorimetric assay where
Dpi 7
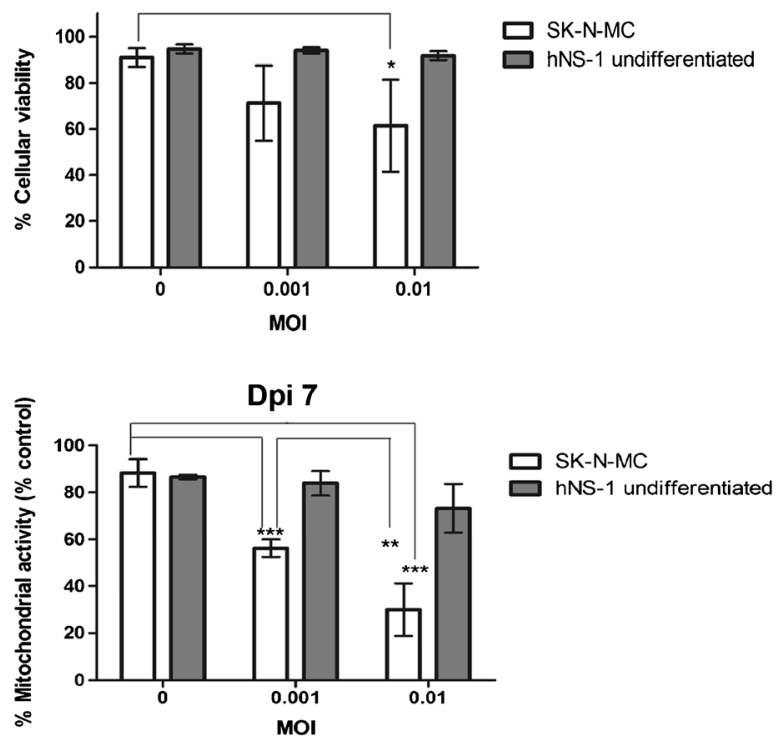

metabolically active cells convert a tetrazolium salt to formazan, and then, the absorbance at $490 \mathrm{~nm}$ was recorded (mean percentage $\pm \mathrm{SD}, n=$ at least three independent experiments). The differences were analyzed for statistical significance using one-way ANOVA $(* p<0.05, * * p<0.01$, $* * * p<0.001)$ 
proliferation and not only in metabolic activity, we also evaluated this parameter by CFSE dilution in cells infected with the MOI of 0.01 and mock-infected cells. However, in infected SK-N-MC cells, we only found an increase in proliferation at 4 dpi and any evident effect at 1 or $7 \mathrm{dpi}$. As the MTS/PMS assay showed, the metabolic activity was higher at $1 \mathrm{dpi}$ and results in cellular division at $4 \mathrm{dpi}$ as it is revealed in CFSE assay; nevertheless, at $7 \mathrm{dpi}$, the cellular activity was diminished, but it does not affect proliferation (Fig. 4a). On the other hand, an enhanced proliferation was evidenced in hNS-1 cells at 4 and $7 \mathrm{dpi}$, although no effect was observed in the MTS/ PMS assay (Fig. 4b, c). This may be related to the lesser susceptibility of these cells to the infection because after 4 dpi, the effects start to emerge.

Differentiation of hNS-1 cells makes them more susceptible to HCMV infection

Since SK-N-MC has a more committed fate than hNS-1 cells, it may explain their distinct susceptibility, and given that hNS1 cell line is multipotent, it can be induced to differentiate and analyze if the differentiation degree modifies their capacity to be infected and the effects of infection. Subsequently, hNS-1 cells were induced to differentiate during 5 days, in order to produce some neural and glial progenitors, and then, they were infected with HCMV at MOI of 0.01 and 0.1 or mockinfected. At $7 \mathrm{dpi}$, it became evident that the CPE was more pronounced in the differentiated cells, and we noticed syncytia formation, some cytoplasmatic inclusions, and vacuoles (Fig. 5a). Differentiated hNS-1 cells begin to express MAP-
$2(6.6 \pm 4.5 \%)$ and GFAP $(4.9 \pm 2.3 \%)$, cellular markers of neurons and astrocytes, respectively (Fig. 5b). At this time point, the cells were infected and the percentage of cells expressing gB were higher $(20.6 \pm 4.6 \%, n=3, p<0.01)$ than in the undifferentiated cells (Fig. 5c) with the MOI of 0.1 at $7 \mathrm{dpi}$. In contrast with the undifferentiated hNS-1, the viability of the differentiated hNS- 1 cells decreases with the progression of HCMV infection even at $1(72.5 \pm 15 \%)$ and $7 \mathrm{dpi}$ $(61.5 \pm 6.7 \%)$ (Fig. $5 \mathrm{~d}$ ). Whereas, the metabolic activity of these infected cells decreases at $1 \mathrm{dpi}(74.1 \pm 6.5 \%, p<0.05)$ and the effect is reverted at $7 \mathrm{dpi}$ (Fig. 5e). Similarly, these effects were observed in the proliferation assay, and we found a decline in cellular division at $1 \mathrm{dpi}(5 \pm 7.4 \%)$, but this effect is lost according to the infection progress (Fig. $5 \mathrm{f}$ ). Indeed, we did not expect a strong effect in proliferation, since these cells were differentiating and only a remaining percentage continues in cell division.

\section{Discussion}

In congenital HCMV infection, the brain is the main infection target and may cause significant damage to the developing brain. It has been recently described that the most susceptible cells inside the CNS are located in the ventricular and subventricular zones containing NPCs (Cheeran et al. 2009; Tsutsui 2009). Infection of NPCs may cause malformations such as microcephaly and cerebellar hypoplasia and functional disorders like mental retardation and epilepsy (Tsutsui
Fig. 4 Proliferation of NPC increases slightly during infection. a SK-N-MC and b hNS-1 cells were incubated in the dark with CFSE $20 \mu \mathrm{M}$ for $10 \mathrm{~min}$; then, the cells were infected with the MOI of 0.01 or mock-infected. NPCs were collected at 1, 4, and $7 \mathrm{dpi}$, and the quantification of CFSE charge was analyzed by flow cytometry (median $\pm \mathrm{IQR}, n=4,{ }^{* *} p<0.01$, $* * * p<0.001$ Mann Whitney U test). c Representative histograms of hNS-1 cells indicating the percentage of cellular division at each time of infection for cells infected with MOI 0.01 and mock-infected. The median percentage is indicated in each plot
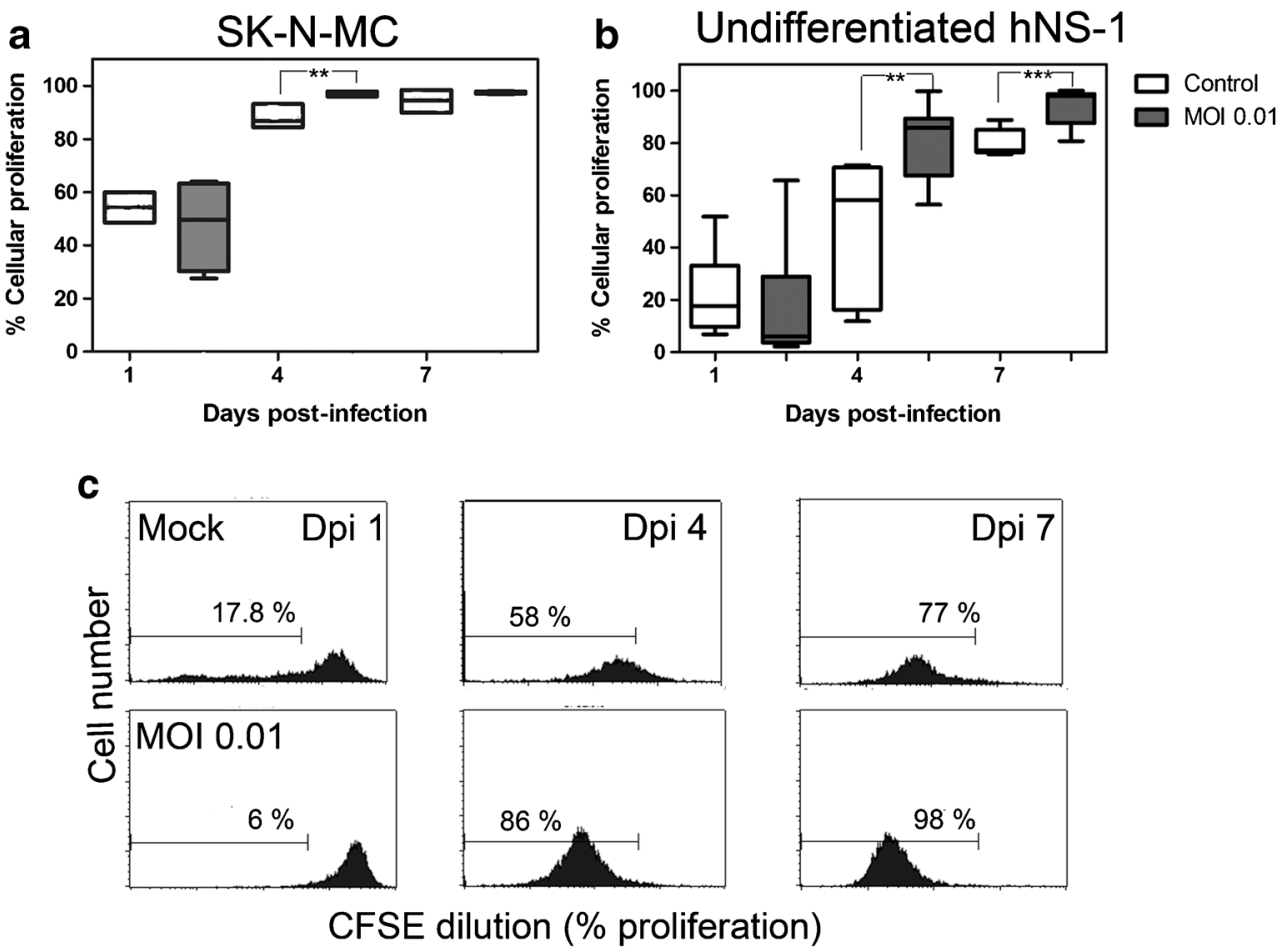
Fig. 5 Differentiation of hNS-1 cells makes them more susceptible to HCMV infection. hNS-1 cells were induced to differentiate during 5 days; then, they were infected with HCMV at MOI of 0.01 and 0.1 or mock-infected. a Differentiated and undifferentiated hNS-1 cells infected with HCMV at 7 dpi showed CPE that was more notable in differentiated cells (cytoplasmic inclusions shown by arrow heads and vacuoles shown by asterisks). b Differentiated hNS-1 cells begin to express MAP-2 and GFAP, cellular markers of neurons and astrocytes, respectively. Scale bar, $50 \mu \mathrm{m}$. c Expression of gB in differentiated hNS-1 cells at $7 \mathrm{dpi}$ (mean percentage $\pm \mathrm{SD}, n=$ at least three independent experiments, $* * p<0.01 t$ test). d In contrast with the undifferentiated hNS-1, the viability of the differentiated hNS- 1 cells decreases with the progression of HCMV infection (mean percentage $\pm \mathrm{SD}, n=$ $4,{ }^{*} p<0.05$ t test). e By MTS/ PMS assay, it was determined that the metabolic activity of these infected cells decreases at $1 \mathrm{dpi}$ and the effect is null at $7 \mathrm{dpi}$ (mean percentage $\pm \mathrm{SD}, n=5,{ }^{*} p<0.05 \mathrm{t}$ test). $\mathbf{f}$ In the proliferation assay using CFSE, we found a decline in cellular division at $1 \mathrm{dpi}$, but this effect is lost according to the infection progress (median $\pm \mathrm{IQR}$, $n=4, * p<0.05$ Mann Whitney U test)
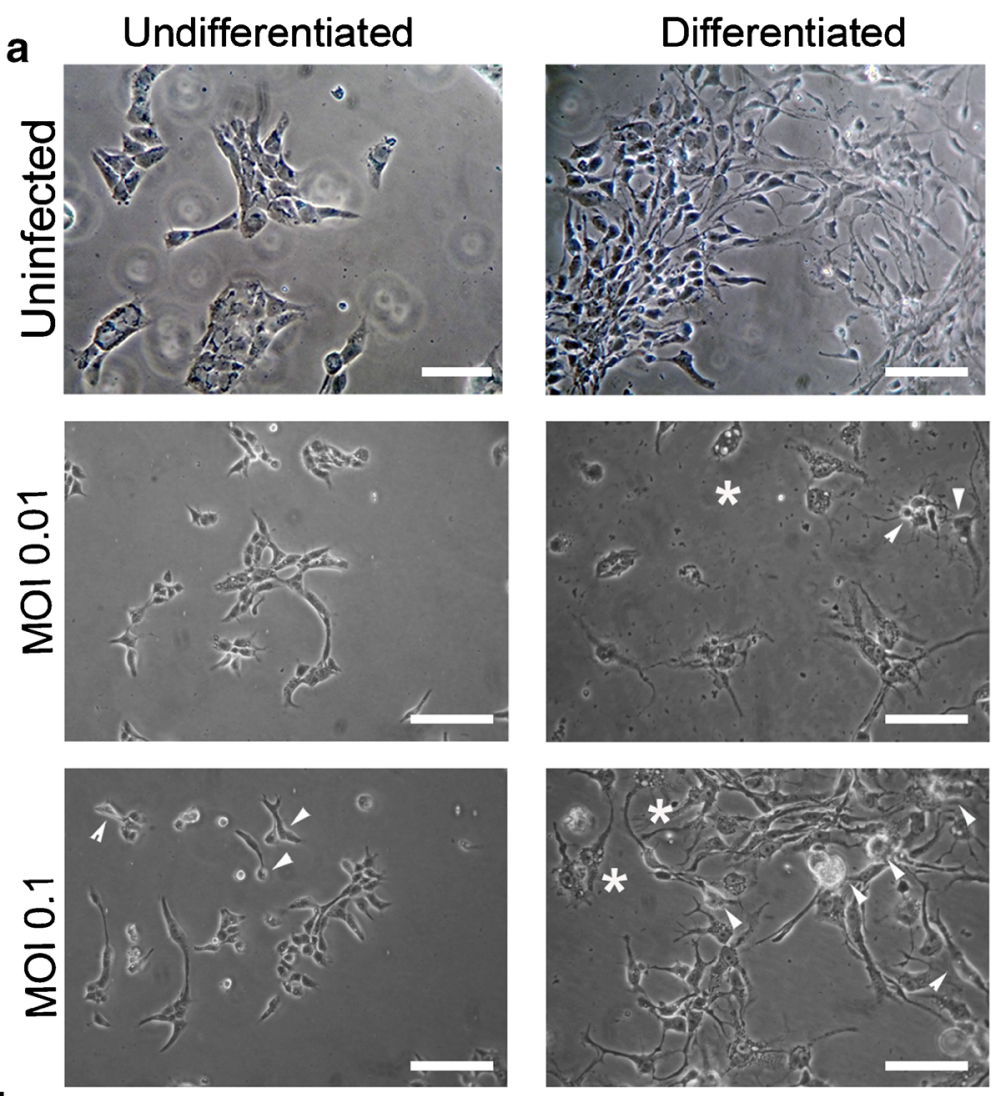

b
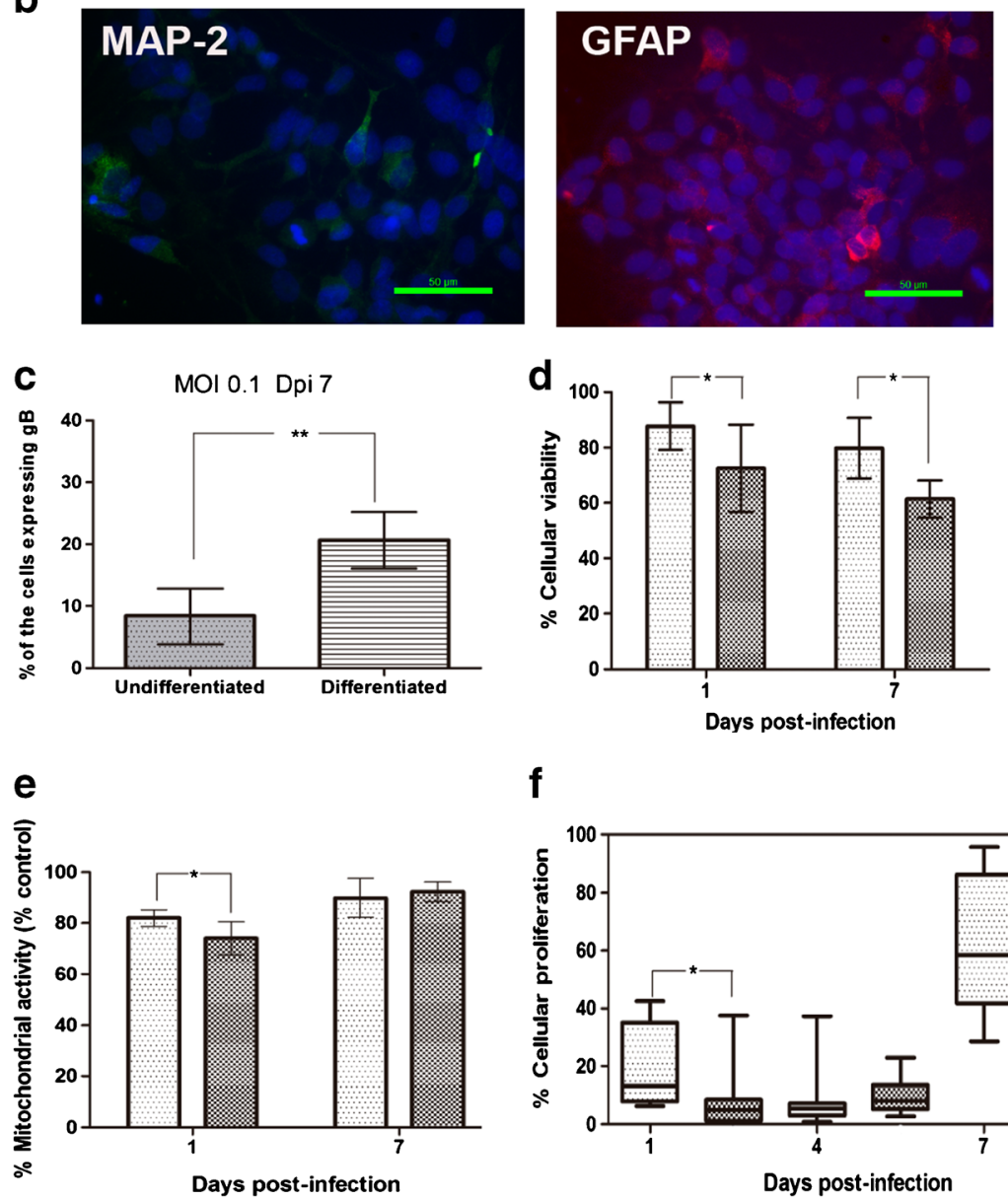

\section{f}

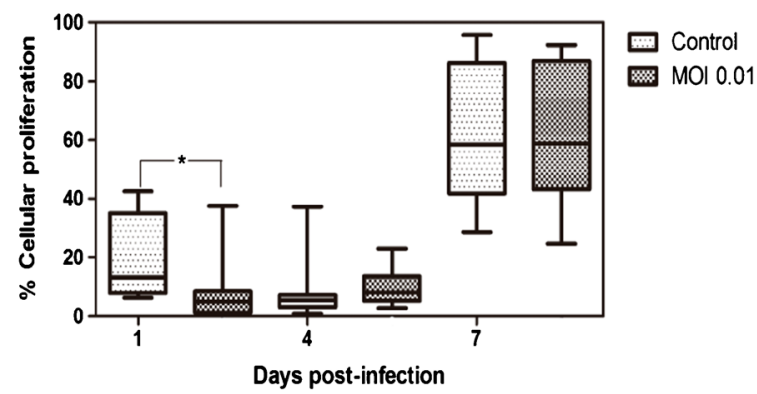


2009). The molecular mechanisms underlying pathogenic responses to HCMV infection of NPCs are poorly understood. Increasing knowledge about how HCMV interferes with the normal function of NPC is therefore of considerable interest because of NPC's role during development. Here, we have used an in vitro model based on human NPC infection to investigate these subjects. We employed the human cell lines SK-N-MC and hNS-1 as a source of NPC. The SK-N-MC is a naturally immortal cell line because it derives from a neuroblastoma, while hNS-1 cell line was genetically perpetuated by transfection of the avian oncogen v-myc and it is conditionally immortalized since it has a strict requirement for mitogens EGF and FGF-2 (Villa et al. 2000). The SK-N-MC cell line is more committed to the neuronal fate than hNS-1 cells which are multipotent and can produce neurons and glia through differentiation (Villa et al. 2000). We confirmed HCMV infection in these cell lines and analyzed cellular viability and proliferation. In SK-N-MC cells, we found out that low viral doses stimulated proliferation, increased their metabolic activity, and induced differentiation; we also noticed that CPE is dose-dependent in this cell line. In contrast, at the same MOIs, hNS-1 cells could only be infected to a lower extent. Scarce evidence of the infection was obtained since the immunoreactivity for gB of HCMV inside the cells was much weaker than in SK-N-MC cells. In addition, no evidence of cell death or modifications in metabolic activity were found, although the proliferation rate increased at late days after infection. Interestingly, after 5 days of differentiation, hNS-1 cells were highly infected showing thus an increased susceptibility. The CPE was more pronounced, and viability, mitochondrial activity, and proliferation decreased while a higher percentage of cells were found infected. These results suggest that neurotoxicity mechanisms associated to HCMV infection may be dependent on the differentiation stage of these NPCs.

The observation of syncytia formation after HCMV infection in SK-N-MC with the MOI of 0.1 is in agreement with earlier reported findings (Luo et al. 2008). However, the temporal course of these changes was different, which could be attributed to HCMV doses. Syncytia formation has also been reported in other cellular types, and it seems to be related to cell fusion induced by viral glycoproteins present in the plasma membrane (Belec et al. 1990; van Den Pol et al. 1999; Kinzler and Compton 2005). On the other hand, when we infected SK$\mathrm{N}-\mathrm{MC}$ with the MOI of 0.01 , cellular prolongations were observed suggesting cellular differentiation to a neuron-like morphology, an unexpected finding since we did not add any other stimulus that may account for these changes, and this normally does not occur in an spontaneous way (Higgins et al. 2009). This induction of differentiation is in agreement with the recent observation of abnormal, spontaneous, and premature differentiation of NPC induced by HCMV (Odeberg et al. 2006; Luo et al. 2010). The virus concentrations that we used were much lower than those in previous studies, what may explain the delayed effect observed. In contrast with those reports, we monitored the CPE in the cells for a more prolonged period of time and observed syncytia formation as the infection progressed. Altogether, these observations suggest that cellular differentiation is stimulated in early stages of infection and that cells tend to fuse forming syncytia as viral concentration increases or time after infection progresses. A possible explanation for this latter effect can be a distinct regulation of transcription factors such as SOX2 which is related to embryonic development and stem cell self-renewal and expression of some receptors like EGFR as well as cytoskeleton components (Jafferji et al. 2009; Luo et al. 2010). Nevertheless, the relevance of HCMV-induced differentiation in the context of CNS development and finding why it is reversed when viral concentration increases are a matter for further investigation. In contrast with our findings in SK-N-MC cells, previous reports showed a decrease in NPC differentiation to neurons and glia after HCMV infection (Odeberg et al. 2006, 2007). Indeed, the experimental methods used in these studies were different, and since we did not induce differentiation, it occurred spontaneously. In addition, the virus concentrations employed were higher in the studies of Odeberg et al. than the ones used in the present work. In the case of hNS-1, we noticed a slight CPE with some owl's eyes and syncytia formation at advanced stages post-infection, indicating that these cells are less susceptible to HCMV infection which may be a consequence of their fully undifferentiated state (hNS-1 is composed of neural stem cells).

Viral particle production was monitored by the viral production assay, through the increased presence of viral DNA in the cell supernatants at different times post-infection. The level of detection of viral particles in the cell lines used here was consistent with the results obtained using a different source of NPC (Luo et al. 2008). However, it is dissimilar to those of others who have reported that NPCs are fully permissive to the infection, but few viral particles are released (Odeberg et al. 2006). We observed that viral production in SK-N-MC was similar to the one in fibroblasts but not detectable in hNS-1. This might reflect the variability of results obtained when working with different NPC cultures. On the one hand, SKN-MC cells may resemble one cell type used (Luo et al. 2008) whereas hNS-1 may be more similar to the other one (Odeberg et al. 2006). Also, when we studied gB expression, we found the majority of SK-N-MC cells being positive in immunocytochemistry; however, only a low percentage of hNS-1 cells were positively stained for $\mathrm{gB}$. This might reflect their low susceptibility to infection and may explain that viral production could not be detected. Since gB is a product of late viral genes, its presence reflects the capacity of the virus and cells for viral replication.

HCMV infection induces cell death of SK-N-MC cells but not of hNS-1 ones. Cell death induced by HCMV could be the result of a necrotic or an apoptotic process since this assay 
does not indicate the involved mechanism (Cheeran et al. 2005; Odeberg et al. 2006). In addition, proliferation assays showed a strong reduction in SK-N-MC proliferation at 7 dpi with both HCMV concentrations. However, paradoxically, at $1 \mathrm{dpi}$, an increased metabolism was observed after HCMV infection; this observation could also derive from an increase in cellular activity due to viral stimulation of macromolecule synthesis during viral replication, rather than an actual increase in the number of cells. Indeed, in the proliferation assay using CFSE, we only detected an increase in cellular division at $4 \mathrm{dpi}$. It is consistent with a previous report of no change in proliferation at 7 dpi using the MOI of 0.1 by the detection of PCNA, a marker for proliferation; however, we showed a previous contradictory induction of proliferation and metabolic activity (Odeberg et al. 2007). It is believed that viruses avoid cellular macromolecule production to elude immune system activation. However, in contrast with other viruses, it has been observed that HCMV stimulates cellular DNA, RNA, and protein synthesis, perhaps because HCMV requires a protein that cannot be produced by HCMV (DeMarchi 1983; Griffiths and Grundy 1987). As expected, cellular metabolism decreased at 7 dpi because although immediate-early proteins stimulate cellular function, early proteins interrupt this process (Griffiths and Grundy 1987). In contrast, hNS-1 cells appeared more resistant even than fibroblast in this MTS/PMS assay and did not show any change in cellular viability, consistent with the lack of viral production, as evidenced by DNA detection. Nevertheless, even though some changes in metabolic activity were detected, cellular proliferation increased at 4 and 7 dpi. This may represent a delayed effect which may be a product of the low rate of infection of these cells. The reduced susceptibility to HCMV infection may be a matter of cellular differentiation since hNS-1 cells can be considered more undifferentiated cells than SK-N-MC ones. Of note, some reports have shown that embryonic stem cells are resistant to HCMV infection and that embryos acquire susceptibility during development (Tsutsui 2009). In order to test this hypothesis, hNS-1 cells were differentiated for 5 days and they were HCMV-infected. At this stage, some cells begin to express GFAP and MAP-2, cellular markers of astroglia and neurons, respectively. Interestingly, a more marked CPE was evidenced in differentiated cells, and cytomegalic inclusions, syncytia formation, vacuoles, and granulations were clearer. A higher percentage of dead cells were found in the viability assay even at 1 and $7 \mathrm{dpi}$, and the positivity for $\mathrm{gB}$ increased in comparison with nondifferentiated hNS-1 cells.

The increased susceptibility to infection after differentiation induction may be a consequence of changes in one or more steps of the infection process, such as attachment, entry, trafficking, nuclear entry, and promoter activity (Kawasaki et al. 2011). It has been reported that the susceptibility of mouse stem cells to CMV may be determined by the concentration of heparan sulfate, presence of $\beta 1$-integrin and vimentin, and nuclear pore density (Kawasaki et al. 2011). It is possible that one of these factors may be related to the acquisition of susceptibility to HCMV in human cells, including hNS-1 cells, which will be the object of further investigation. Interestingly, as the mouse embryonic stem cells are refractory to the infection, differentiated neurons also have shown an apparent refractivity (Cheeran et al. 2009). This effect has been suggested to be explained by the expression of the transcription factor C/EBP $\beta$ and its dominant negative isoform which binds to the enhancer region and inhibits transcription from the HCMV MIEP (Cheeran et al. 2005). By this reason, we assessed the role of differentiation in hNS-1 cells at an early stage when some progenitors begin to differentiate and not when the differentiated neurons can be found.

Because our main objective was to describe the earliest effects of HCMV infection, we have used significantly lower MOI than those employed in other reports (Cheeran et al. 2005; Odeberg et al. 2006, 2007; Luo et al. 2008, 2010). Instead of showing the deepest impact of the infection, we decided to monitor the progression in different time points, in order to follow slight effects. This scheme allows us to show that during a congenital infection, even when a low viral dose reaches the CNS, it eventually may be able to produce effects in the NPC.

The mechanisms that produce CNS damage during congenital HCMV infection have not been clearly elucidated. However, recent efforts through in vivo and in vitro models have allowed a better understanding of the pathogenesis of this viral infection. It is well known that the major neurological damage occurs during intrauterine infection by HCMV at early pregnancy stages. In addition, some brain localizations like ventricular and subventricular zones are particularly important; therefore, it is possible that NPCs play a substantial role during neurological damage. Previous studies have shown that CMV infection in NPC results in differentiation inhibition, reduced proliferation, cellular signaling interference, and cell death; together, these effects may produce migration defects during brain development (Kawasaki and Tsutsui 2003; Cheeran et al. 2005; Odeberg et al. 2006; Ho and van den Pol 2007). We developed an in vitro model using two different NPCs promoting the use of these cell lines instead of primary cultures. Permissibility to infection and diminished cellular viability were corroborated in the most differentiated cell line; however, in contrast with other studies, we found out that low viral concentrations induced cellular proliferation in a transitory way in addition to possible differentiation stimulation. Also, we verified that the permissibility to HCMV infection is closely related to cellular differentiation since cells that more closely resemble neural stem cells were less vulnerable to HCMV and they acquired susceptibility through differentiation.

In conclusion, these experimental results can be interpreted in the context of the observed abnormalities during a 
congenital infection. It is important to underline the importance of NPC susceptibility to HCMV because infection of these cells may have a significant effect on fetal brain development. Thus, both cell death and spontaneous and premature differentiation of NPC can affect the normal proliferation and migration patterns of neurons during fetal development leading to altered brain architecture and function. Therefore, in order to elucidate the mechanisms and clinical consequences of the observed effects, further investigations are required.

Acknowledgments This work was supported by grant from CONACYT (CB16782 and \#120452), PROMEP (103.5/10/7697), and FAI-UASLP (C12-FAI-03-62.62). Hilda Gonzalez and Claudia Salazar were recipients of scholarships from CONACYT (CVU 265757 and 265189).

Conflict of interests The authors declare that there is no conflict of interests regarding the publication of this paper.

\section{References}

Agol VI (2012) Cytopathic effects: virus-modulated manifestations of innate immunity? Trends Microbiol 20(12):570-576

Belec L, Mhiri C, Belghiti D, Geny C, Boudes P, Gray F (1990) Cytomegalovirus (CMV) and human immunodeficiency virus (HIV) co-infection, of multinucleated giant cells in acquired immunodeficiency syndrome (AIDS) encephalopathy. Arch Anat Cytol Pathol 38:189-197

Cheeran MC, Hu S, Ni HT, Sheng W, Palmquist JM, Peterson PK, Lokensgard JR (2005) Neural precursor cell susceptibility to human cytomegalovirus diverges along glial or neuronal differentiation pathways. J Neurosci Res 82:839-850

Cheeran MC, Lokensgard JR, Schleiss MR (2009) Neuropathogenesis of congenital cytomegalovirus infection: disease mechanisms and prospects for intervention. Clin Microbiol Rev 22:99-126, Table of Contents

DeMarchi JM (1983) Nature of the block in the expression of some early virus genes in cells abortively infected with human cytomegalovirus. Virology 129:287-297

Griffiths PD, Grundy JE (1987) Molecular biology and immunology of cytomegalovirus. Biochem J 241:313-324

Higgins S, Wong SH, Richner M, Rowe CL, Newgreen DF, Werther GA, Russo VC (2009) Fibroblast growth factor 2 reactivates G1 checkpoint in SK-N-MC cells via regulation of $\mathrm{p} 21$, inhibitor of differentiation genes (Id1-3), and epithelium-mesenchyme transition-like events. Endocrinology 150:4044 4055

Ho M (2008) The history of cytomegalovirus and its diseases. Med Microbiol Immunol 197:65-73

Ho WS, van den Pol AN (2007) Bystander attenuation of neuronal and astrocyte intercellular communication by murine cytomegalovirus infection of glia. J Virol 81:7286-7292

Jafferji I, Bain M, King C, Sinclair JH (2009) Inhibition of epidermal growth factor receptor (EGFR) expression by human cytomegalovirus correlates with an increase in the expression and binding of Wilms' Tumour 1 protein to the EGFR promoter. J Gen Virol 90: $1569-1574$

Kawasaki H, Tsutsui Y (2003) Brain slice culture for analysis of developmental brain disorders with special reference to congenital cytomegalovirus infection. Congenit Anom (Kyoto) 43:105-113
Kawasaki H, Kosugi I, Arai Y, Iwashita T, Tsutsui Y (2011) Mouse embryonic stem cells inhibit murine cytomegalovirus infection through a multi-step process. PLoS One 6:e17492

Kinzler ER, Compton T (2005) Characterization of human cytomegalovirus glycoprotein-induced cell-cell fusion. J Virol 79:7827-7837

Köhler TH (1995) Quantitative RT-PCR. In: Quantitation of mRNA by polymerase chain reaction (Springer-Verlag, ed), pp 71-80 Berlin Heidelberg

Luo MH, Schwartz PH, Fortunato EA (2008) Neonatal neural progenitor cells and their neuronal and glial cell derivatives are fully permissive for human cytomegalovirus infection. J Virol 82:9994-10007

Luo MH, Hannemann H, Kulkarni AS, Schwartz PH, O’Dowd JM, Fortunato EA (2010) Human cytomegalovirus infection causes premature and abnormal differentiation of human neural progenitor cells. J Virol 84:3528-3541

Martinez-Serrano A, Rubio FJ, Navarro B, Bueno C, Villa A (2001) Human neural stem and progenitor cells: in vitro and in vivo properties, and potential for gene therapy and cell replacement in the CNS. Curr Gene Ther 1(3):279-299

Matsukage S, Kosugi I, Kawasaski H, Miura K, Kitani H, Tsutsui Y (2006) Mouse embryonic stem cells are not susceptible to cytomegalovirus but acquire susceptibility during differentiation. Birth Defects Res A Clin Mol Teratol 76:115-125

Monsivais-Urenda A, Noyola-Cherpitel D, Hernandez-Salinas A, GarciaSepulveda C, Romo N, Baranda L, Lopez-Botet M, GonzalezAmaro R (2010) Influence of human cytomegalovirus infection on the NK cell receptor repertoire in children. Eur J Immunol 40:1418 1427

Navarro-Galve B, Villa A, Bueno C, Thompson L, Johansen J, MartinezSerrano A (2005) Gene marking of human neural stem/precursor cells using green fluorescent proteins. J Gene Med 7(1):18-29

Noyola DE, Jimenez-Capdeville ME, Demmler-Harrison GJ (2010) Central nervous system disorders in infants with congenital cytomegalovirus infection. Neurol Res 32:278-284

Odeberg J, Wolmer N, Falci S, Westgren M, Seiger A, Soderberg-Naucler C (2006) Human cytomegalovirus inhibits neuronal differentiation and induces apoptosis in human neural precursor cells. J Virol 80: 8929-8939

Odeberg J, Wolmer N, Falci S, Westgren M, Sundtrom E, Seiger A, Soderberg-Naucler C (2007) Late human cytomegalovirus (HCMV) proteins inhibit differentiation of human neural precursor cells into astrocytes. J Neurosci Res 85:583-593

Ornoy A (2007) Fetal effects of primary and non-primary cytomegalovirus infection in pregnancy: are we close to prevention? Isr Med Assoc J 9:398-401

Rubio FJ, Bueno C, Villa A, Navarro B, Martinez-Serrano A (2000) Genetically perpetuated human neural stem cells engraft and differentiate into the adult mammalian brain. Mol Cell Neurosci 16:1-13

Shinmura Y, Kosugi I, Aiba-Masago S, Baba S, Yong LR, Tsutsui Y (1997) Disordered migration and loss of virus-infected neuronal cells in developing mouse brains infected with murine cytomegalovirus. Acta Neuropathol 93:551-557

Tsutsui Y (2009) Effects of cytomegalovirus infection on embryogenesis and brain development. Congenit Anom (Kyoto) 49:47-55

Tsutsui Y, Kawasaki H, Kosugi I (2002) Reactivation of latent cytomegalovirus infection in mouse brain cells detected after transfer to brain slice cultures. J Virol 76:7247-7254

Tsutsui Y, Kosugi I, Kawasaki H, Arai Y, Han GP, Li L, Kaneta M (2008) Roles of neural stem progenitor cells in cytomegalovirus infection of the brain in mouse models. Pathol Int 58:257-267

van Den Pol AN, Mocarski E, Saederup N, Vieira J, Meier TJ (1999) Cytomegalovirus cell tropism, replication, and gene transfer in brain. J Neurosci 19:10948-10965

Villa A, Snyder EY, Vescovi A, Martinez-Serrano A (2000) Establishment and properties of a growth factor-dependent, 
perpetual neural stem cell line from the human CNS. Exp Neurol 161:67-84

Villa A, Rubio FJ, Navarro B, Bueno C, Martinez-Serrano A (2001) Human neural stem cells in vitro. A focus on their isolation and perpetuation. Biomed Pharmacother 55:91-95

Villa A, Navarro B, Martinez-Serrano A (2002) Genetic perpetuation of in vitro expanded human neural stem cells: cellular properties and therapeutic potential. Brain Res Bull 57:789794
Villa A, Navarro-Galve B, Bueno C, Franco S, Blasco MA, MartinezSerrano A (2004) Long-term molecular and cellular stability of human neural stem cell lines. Exp Cell Res 294:559-570

Wentworth BB, French L (1970) Plaque assay of cytomegalovirus strains of human origin. Proc Soc Exp Biol Med 135:253-258

Woolf NK, Jaquish DV, Koehrn FJ (2007) Transplacental murine cytomegalovirus infection in the brain of SCID mice. Virol J 4:26

Wynn KK, Khanna R (2006) Models of CMV infection. Drug Discov Today 3:91-96 